\title{
La Red de Viveros de Plantas Nativas de Argentina (REVINA): Una perspectiva etnobotánica para fortalecer la restauración de ecosistemas en la Argentina
}

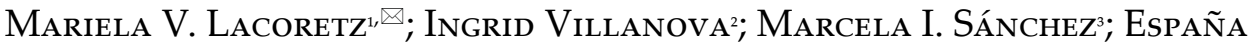 \\ Verrastro* \& Piedad M. Cristiano ${ }^{1}$
}

\author{
${ }^{1}$ Facultad de Ciencias Exactas y Naturales, Universidad de Buenos Aires, IEGEBA-CONICET. CABA. ${ }^{2}$ Centro de \\ Investigación y Desarrollo Tecnológico para la Agricultura Familiar, INTA. Buenos Aires. ${ }^{3}$ Jardín Botánico Arturo E. \\ Ragonese, Instituto de Recursos Biológicos, INTA. Buenos Aires. ${ }^{4}$ Coordinación de Programas Socioambientales, Secretaría de \\ Extensión, Universidad Nacional de San Martín. Buenos Aires.
}

Resumen. La Red de Viveros de Plantas Nativas de Argentina (REVINA) vincula a diferentes actores sociales involucrados en producir plantas nativas, en ponerlas en valor y en restaurar los ecosistemas de la Argentina. Fortalecer la red es fundamental para cumplir con las metas de restauración del país, teniendo en cuenta que la comunidad de viveristas generará las especies de plantas que serán los insumos primordiales de los proyectos de restauración. En este trabajo se estudió la red de viveros de plantas nativas, la comunidad de viveristas y las plantas que éstos producen, con el fin de fortalecer los proyectos de restauración en nuestro país a través de relevar los participantes de los encuentros anuales de la REVINA entre 2016 y 2020. Observamos que la red ha crecido notablemente desde su creación, lo cual refleja que el interés por las plantas nativas ha aumentado en los últimos años. En su mayoría, las especies vegetales que se cultivan en los viveros pertenecen a la familia Fabaceae, son leñosas, y presentan un sesgo fuerte hacia especies del centro-este del país, en consonancia con el número de viveros que se concentra en esa región. La mayor parte de los viveros tiene una producción pequeña, aunque a lo largo de los años se sumaron viveros productores de mayor tamaño. La perspectiva utilizada permitió obtener una primera lista de viveros de plantas nativas y de las especies que éstos cultivan. Finalmente, se proponen algunos aspectos a mejorar para fortalecer esta red de actores y así cumplir con las iniciativas de restauración del país.

[Palabras clave: restauración ecológica, producción de plantas, ciencia socialmente robusta, co-producción del conocimiento]

Abstract. The Argentine Native Plant Nursery Network (REVINA): An ethnobotanical perspective to strengthen ecosystem restoration in Argentina. The Argentine Native Plant Nursery Network (REVINA) links different social actors involved in native plant production, their valorization and the restoration of Argentina's ecosystems. It is essential to strengthen the network in order to meet the country's restoration goals, considering that the nursery community will generate the plant species that will be the primary inputs for restoration projects. In this paper we study the native plant nursery network, the nursery community and the plants they produce, in order to strengthen restoration projects in Argentina through the survey of the participants of the annual meetings of the REVINA in the period 2016-2020. We observed the network has grown remarkably since its creation, reflecting the increasing interest in native plants in recent years. Most of the plant species grown in the nurseries belonged to the Fabaceae family, are woody, and show a strong bias towards species from the centre-east of the country, in line with the number of nurseries concentrated in that region. A great number of nurseries have a small production, although, over the years, larger nurseries joined the network. The perspective used allowed us to obtain a first list of native plant nurseries and the species they cultivate. Finally, some aspects are proposed in order to strengthen this network of actors and thus be able to fulfill the restoration initiatives of the country.

[Keywords: ecological restoration, plant production, socially robust science, knowledge co-production]

Editora asociada: Roxana Aragón

mariela.lacoretz@gmail.com
Recibido: 3 de Junio de 2021

Aceptado: 20 de Diciembre de 2021 


\section{INTRODUCCIÓN}

La interacción entre las sociedades y las plantas ha modelado históricamente el mundo físico donde las personas habitan y construyen sus realidades (Hamilton et al. 2003). La etnobotánica es la ciencia que estudia la relación entre los seres humanos y su entorno vegetal, y abarca tanto a las ciencias naturales como sociales (Bennett 2005; Arenas and Martínez 2012; Hurrell and Paulino de Albuquerque 2012). Si bien esta ciencia ha sido asociada al estudio del uso de las plantas por pueblos originarios o culturas específicas, en la actualidad se ha propuesto que esta disciplina abarca cualquier relación entre la sociedad y las plantas (Bennett 2005). En la Argentina, en los últimos años se llevaron a cabo diferentes estudios etnobotánicos que abarcaron desde el análisis del uso de las plantas por las comunidades originarias hasta el empleo de las mismas en ambientes urbanos (e.g., Capparelli et al. 2011; Muiño 2011; Arenas 2012; Rovere et al. 2013; Hurrell 2014; Suárez 2014; Rosso and Scarpa 2019). Muchos de estos trabajos resaltan la importancia del conocimiento botánico local, que es el conjunto de saberes y creencias sobre las plantas en un cierto contexto cultural, que puede reflejarse en estrategias de producción de las plantas (Pochettino et al. 2014). En particular, la etnobotánica aplicada se basa en conocimientos populares y científicos, y permite comparar e integrar estas dos fuentes de información en beneficio de la conservación y el desarrollo sostenible (Hamilton et al. 2003). Este enfoque interdisciplinario y participativo se orienta a resolver problemas locales; permite generar inventarios botánicos, aportar a la conservación de plantas nativas y, por ende, a mantener la diversidad biológica en general (Hamilton et al. 2003).

Estudiar, conservar y propagar las plantas nativas es importante desde múltiples enfoques, tanto ecológicos como económicos y culturales (Dumroese et al. 2009; Eynard et al. 2020). En cuanto a su importancia ecológica, en sistemas terrestres, las plantas nativas forman la estructura del hábitat y son la fuente de nutrientes para la mayoría de las formas de vida (Hamilton et al. 2003; Bardgett and Wardle 2010). Por ejemplo, la dieta de la mayoría de las especies de insectos herbívoros se asocia localmente a una única familia o especie de plantas nativas (Forister et al. 2015). Si las plantas nativas de un determinado ecosistema son eliminadas o desplazadas por especies exóticas, esto puede conducir a pérdidas de la biodiversidad (Chapin III et al. 2000). Asimismo, existe una asociación positiva y bien estudiada entre la biodiversidad y los servicios ecosistémicos (Díaz et al. 2006; Harrison et al. 2014). Estos servicios redundan en beneficios económicos y en el bienestar humano (MEA 2005; Gann et al. 2019). En síntesis, no sólo es importante cultivar plantas nativas porque representan parte de nuestra historia, identidad y patrimonio natural, sino también porque cumplen un papel fundamental en sostener los ecosistemas, la biodiversidad y los servicios que éstos proveen.

Muchos de los ecosistemas en la Argentina se encuentran amenazados por los cambios en el uso del suelo, los incendios y la invasión de especies exóticas. Por lo tanto, además de conservarlos es necesario tomar acciones de manejo para su restauración (Pérez et al. 2018) y resulta fundamental producir plantas nativas en vivero (Haase and Davis 2017). Las especies de plantas seleccionadas por los viveristas, que se pueden elegir por aspectos ecológicos o culturales, tendrán un impacto en el resultado del proceso de la restauración ecológica. Estudiar y conocer la identidad de las plantas cultivadas por los viveros de plantas nativas es crucial, ya que serán el insumo para los proyectos de restauración y determinarán la composición de especies que tendrá el ecosistema que se intenta reconstruir. Por lo tanto, los proyectos de restauración se beneficiarían de los resultados de estudios etnobotánicos que describan qué especies cultiva la comunidad de viveristas de plantas nativas.

La Argentina tiene una serie de metas nacionales y compromisos internacionales (Ley Nro. 26331 2007; Bonn Challenge 2011; Initiative 20x20 2014; PNRBN 2019; PPRPA-NB 2020) que implica restaurar miles de hectáreas en la próxima década. Estos proyectos de restauración demandarán un gran volumen de plantas nativas que, como país, aún no estamos preparados para producir (por ejemplo, ver Lacoretz et al. 2021 para la provincia de Buenos Aires). Para satisfacer esta demanda se debe aumentar la producción en los viveros mediante el fortalecimiento de los vínculos institucionales entre distintos actores sociales, a fin de realizar una restauración socialmente robusta con colaboración de toda la sociedad (Nowotny et al. 2001; Gross 2006).

La Red de Viveros de Plantas Nativas de Argentina (REVINA) se inició en el 2016 y 
vincula a distintos actores sociales, como productores, investigadores, paisajistas y docentes, entre otros. Estos actores de la red se encuentran involucrados en la producción de plantas nativas, en su puesta en valor y en la restauración delos ecosistemas dela Argentina. La misión de la REVINA es promover el uso de plantas nativas en la Argentina, impulsando la educación, la investigación, la producción y la comercialización para la restauración ambiental y la planificación del paisaje a todas las escalas, de manera sustentable y sostenible. Entre los objetivos de la red se destaca crear un espacio de cooperación entre las partes mediante el intercambio de información y experiencias de cultivo, generar un marco de diálogo frente a la demanda de plantas nativas, difundir la importancia social, económica y ambiental de las plantas nativas, promover su uso en espacios verdes públicos y privados, apoyar la formación de nuevos viveros, fortalecer los ya existentes y promover la conservación in situ y ex situ. La red es una organización horizontal que posee 14 nodos (CABA, AMBA Norte, AMBA Oeste, AMBA Sur, Buenos Aires Norte, Buenos Aires Cuenca del Salado, Buenos Aires Costa Atlántica, Buenos Aires Tandilia, Buenos Aires SO, Entre Ríos-ROU, Santa Fe, Córdoba, Mendoza y Misiones) y comisiones que se encargan de temas específicos (Educación-InvestigaciónConservación; Viveristas y producción; Paisajismo; Mapeo). Una de las acciones principales de la REVINA es organizar los encuentros anuales; desde sus inicios se llevaron a cabo 5 encuentros.

Fortalecer y hacer crecer la articulación entre los actores sociales que conforman la red es esencial para cumplir con las metas de restauración de la Argentina, teniendo en cuenta quela comunidad de viveristas generará las especies de plantas que serán los insumos primordiales de los proyectos de restauración. Para potenciar la red, primero tenemos que saber cuál es su estado actual. Por ello, en este trabajo estudiamos la red de viveros de plantas nativas, la comunidad de viveristas y las plantas que éstos producen, con el fin de fortalecer los proyectos de restauración en la Argentina a través del relevamiento de los participantes de los encuentros de la REVINA en el período 2016-2020. Este trabajo tuvo como objetivos caracterizar a los integrantes de la REVINA (en cuanto a su actividad e institución de pertenencia) y describir a los viveros que integran la red (considerando ubicación, especies cultivadas y producción). En este estudio se brinda la primera lista de viveros de plantas nativas de la Argentina y de las especies que estos viveros producen a nivel nacional. Esta caracterización también ayudará a dar a conocer esta red, a determinar estrategias de planificación interna para fortalecerla y a resaltar el valor de la misma como generadora de interrelaciones institucionales.

\section{Materiales y Métodos}

Los datos fueron recopilados a partir de los formularios de inscripción a los encuentros anuales entre los años 2016 y 2020. El primer encuentro de la REVINA se realizó en 2016 en la Estación Experimental AMBA del Instituto Nacional de Tecnología Agropecuaria (INTA, Ituzaingó, Buenos Aires). El segundo encuentro se llevó a cabo en 2017 en el Jardín Botánico Arturo E. Ragonese (Instituto de Recursos Biológicos, INTA, Hurlingham, Buenos Aires), con el objetivo de profundizar el diálogo y la cooperación. El tercer encuentro se realizó en 2018 en la Universidad Nacional de Luján (UNLu, Luján, Buenos Aires), y tuvo como motor "empoderar a los viveros para consolidar un perfil productivo-comercial". El cuarto encuentro se realizó en 2019 en la Universidad Nacional de San Martín (UNSAM, San Martín, Buenos Aires), con el lema "Fortalecer los Biocorredores como espacios de restauración ecológica". Por último, en 2020 se realizó el 5to encuentro de la REVINA de manera virtual con el lema "Promoviendo Estrategias de Colaboración entre las Áreas Protegidas y la Red de Viveros de Plantas Nativas".

Se realizó un trabajo de consolidación y unificación de criterios entre las distintas bases de datos, dado que las preguntas de los formularios de inscripción fueron variando año a año dependiendo de la institución organizadora. Las preguntas que se les realizó a los inscriptos incluyeron: 1) localidad de procedencia, 2) actividad (docente, estudiante, investigador, paisajista, particular, productor, funcionario, comerciante), 3) tipo de institución de pertenencia (universidad, instituciones científico-técnicas, gubernamental, organizaciones no gubernamentales-ONG, áreas protegidas, jardines botánicos), 4) posee o no vivero, 5) ubicación del vivero, 6) tipo de vivero (comercial o no comercial), 8) especies de plantas producidas en el vivero, y 7) producción anual de plantas (hasta 400 plantas, de 400 a 1000 plantas, de 1000 a 4000 plantas, de 4000 a 10000 plantas, más de 10000). 
Con la información recolectada, se calculó la frecuencia absoluta de participantes o viveros por año de cada variable, según correspondiera. Los datos espaciales fueron procesados con el programa (QGIS Development Team 2019) $\mathrm{y}$ el resto de las figuras con el programa $\mathrm{R}$ v.4.0.4 (R Core Team 2021). Para elaborar la lista de especies de plantas se reemplazaron los nombres comunes por científicos, y se calculó el porcentaje de viveros que produjo cada especie con respecto al total de viveros. Los datos sobre familia, forma de vida y distribución geográfica de las especies fueron consultados en el Catálogo de las Plantas Vasculares del Conosur, en línea, del Instituto de Botánica Darwinion (Darwinion 2021).

\section{Resultados}

La cantidad de participantes en los encuentros de la REVINA aumentó a lo largo del tiempo (Figura 1). El primer encuentro contó con
103 inscriptos, número que creció de manera progresiva y continua entre 2016 y 2019 en los encuentros de modalidad presencial (Figura 1). En el año 2020, el encuentro se realizó de forma virtual y la participación aumentó 437\% respecto del año anterior (Figura 1). Entre los años 2016 y 2019, estos participantes procedían de entre 5 y 11 provincias de la Argentina, pero en el último año se registraron participantes de todas las provincias del país, concentrándose en la región centro-este (Figura 1). En el año 2020 participaron también personas de otros países de Latinoamérica (Uruguay, Paraguay, Perú, Bolivia, Brasil, México, Costa Rica, Colombia) y del mundo (Estados Unidos, Australia).

La actividad ola profesión de los participantes fue diversa; en su mayoría, docentes y estudiantes, además de investigadores, paisajistas, productores, funcionarios públicos y comerciantes (Figura 2). El año
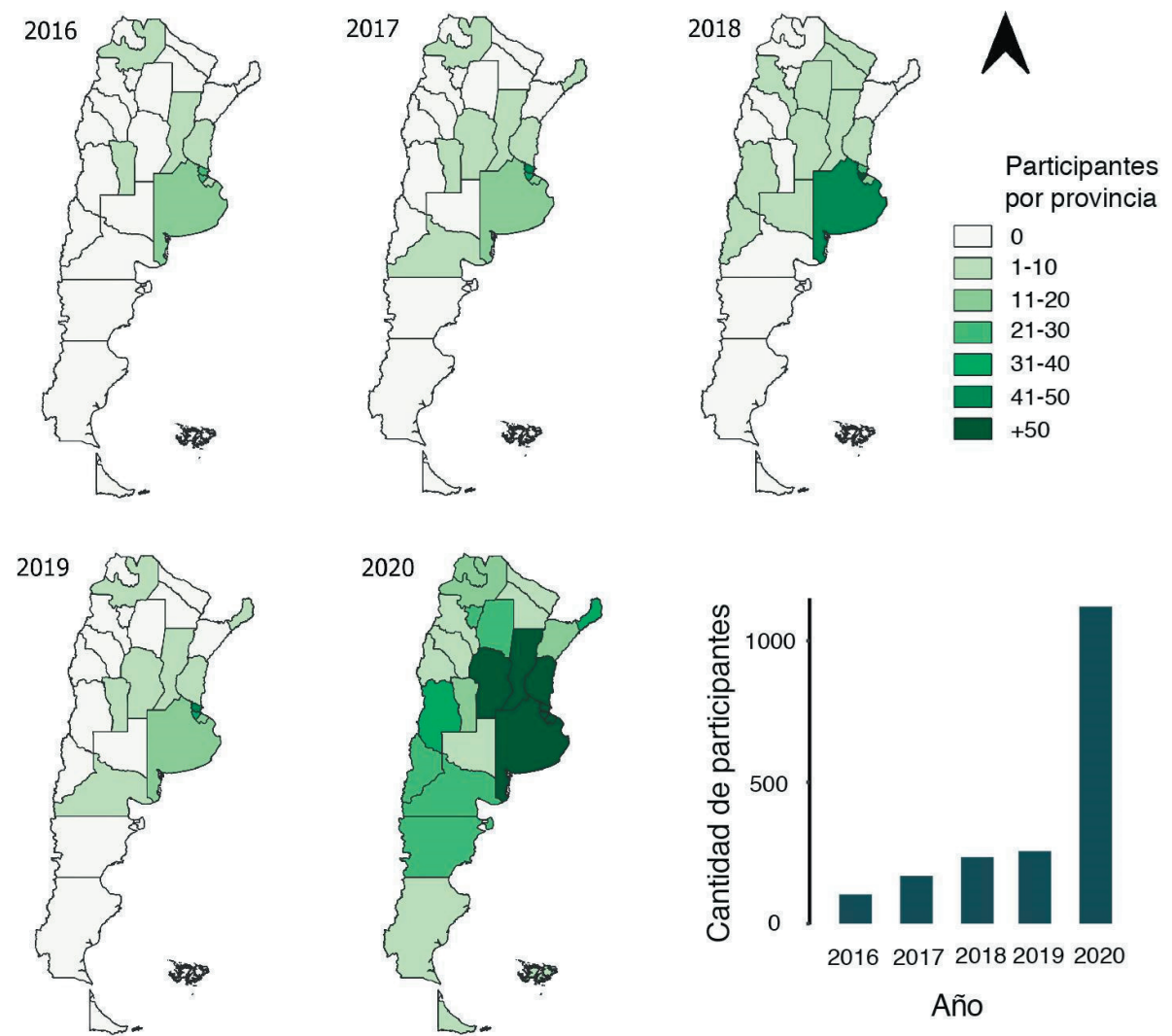

Año

Figura 1. Cantidad de inscriptos por año y provincia entre 2016 y 2020, y total de asistentes en los encuentros de la REVINA (Red de Viveros de Plantas Nativas de Argentina). Entre esos años, la participación aumentó en todo el país, en especial en el último encuentro, que se realizó de forma virtual.

Figure 1. Number of participants per year and province between 2016 and 2020, and total number of attendees at REVINA (Argentine Native Plant Nursery Network) meetings. Between these years, participation increased across the country, especially at the last meeting, which was held virtually. 


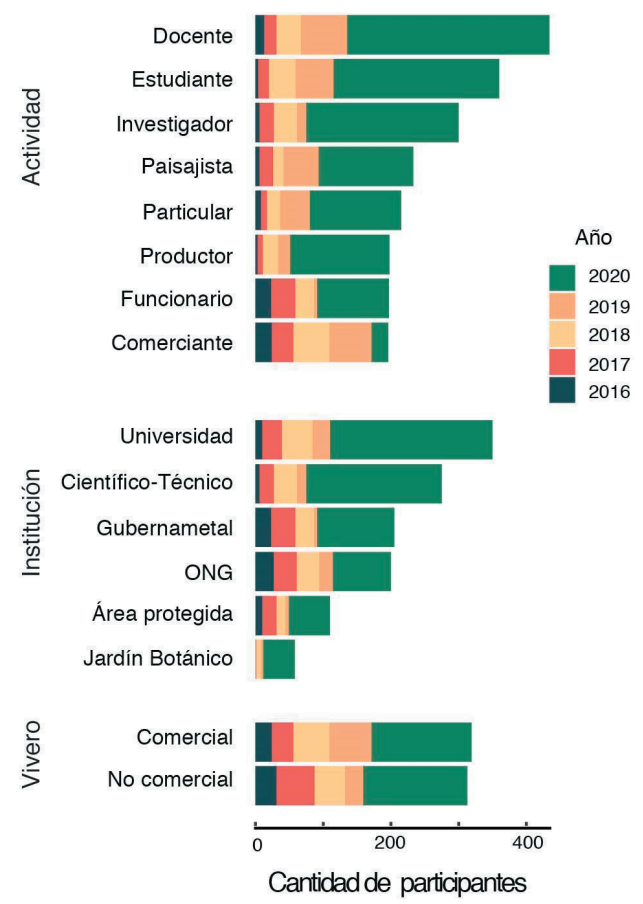

Figura 2. Cantidad de inscriptos por año en los encuentros de la REVINA (Red de Viveros de Plantas Nativas de Argentina) según actividad, institución y tipo de vivero. Figure 2. Number of participants per year in the meetings of REVINA (Argentine Native Plant Nursery Network) according to activity, institution and type of nursery.
2020 fue el de mayor concurrencia para la mayoría de las actividades (Figura 2). Los participantes pertenecieron a diferentes tipos de instituciones, tales como universidades, institutos de ciencia y técnica, organismos de gobierno, organismos no gubernamentales (ONG), áreas protegidas y jardines botánicos (Figura 2). La cantidad de participantes por tipo de institución a lo largo del tiempo no fue constante (Figura 2). Algunos tipos de institución disminuyeron su participación en 2019, por ejemplo, áreas protegidas y ONG, condición que fue revertida en el año 2020 (Figura 2). La proporción de participantes con viveros comerciales y no comerciales fue homogénea a lo largo de los años, y en el 2020 aumentó la concurrencia de ambos tipos de viveristas (Figura 2). Los viveristas con fines comerciales fueron en su mayoría productores, paisajistas y comerciantes, y aquellos que integraban una institución pertenecieron frecuentemente a ONG (Figura 3). Los viveristas con fines no comerciales se dedicaron sobre todo a la docencia, a la investigación o fueron funcionarios públicos y trabajadores de áreas protegidas, y sus instituciones de pertenencia más frecuentes fueron las universidades, los organismos de gobierno, las áreas protegidas y los jardines botánicos (Figura 3).

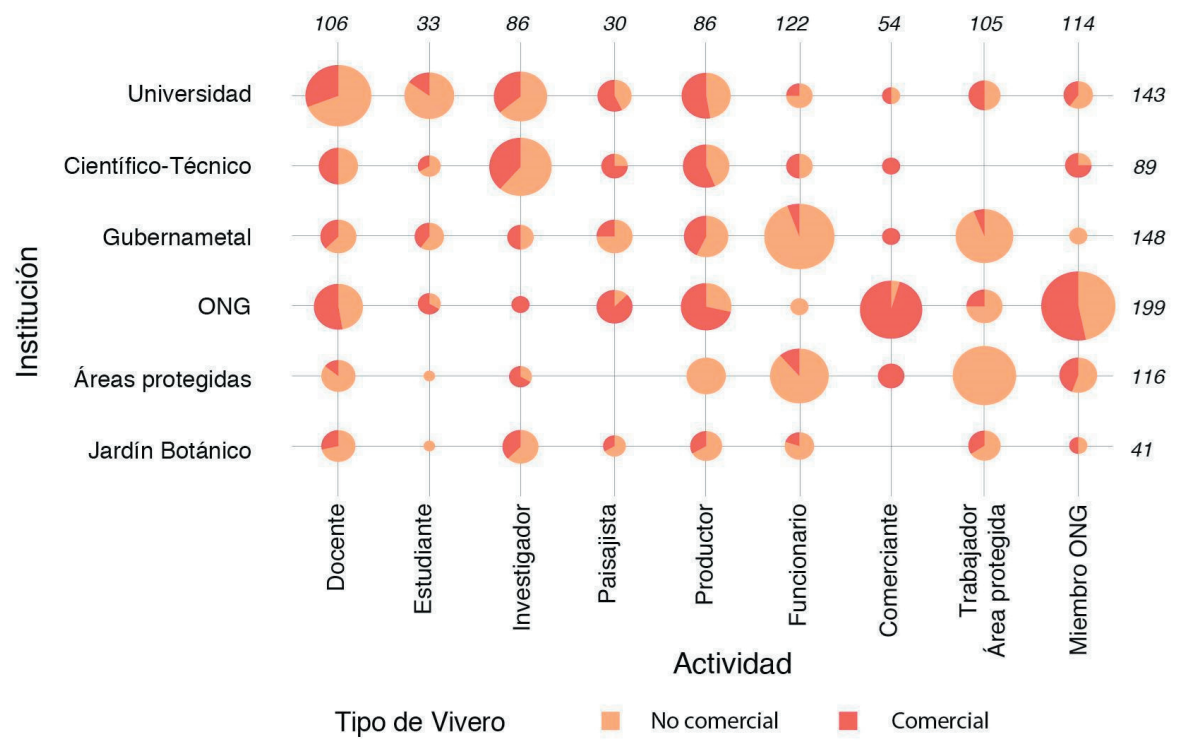

Figura 3. Caracterización de las personas que forman parte de viveros no comerciales y comerciales. Se tuvo en cuenta su pertenencia institucional y su actividad profesional para el período 2016-2020. El radio de los círculos (tamaño) es proporcional, en escala logarítmica, a la cantidad de viveristas. Se indica el número total de participantes para las distintas categorías de las variables Actividad (marginal superior) e Institución (marginal derecho).

Figure 3. Characterization of people who are part of non-commercial and commercial nurseries, taking into account their institutional membership and professional activity for the period 2016-2020. The radius of the circles (size) is proportional, in logarithmic scale, to the number of nursery workers. The total number of participants is indicated for the different categories of the variables Activity (upper marginal) and Institution (right marginal). 


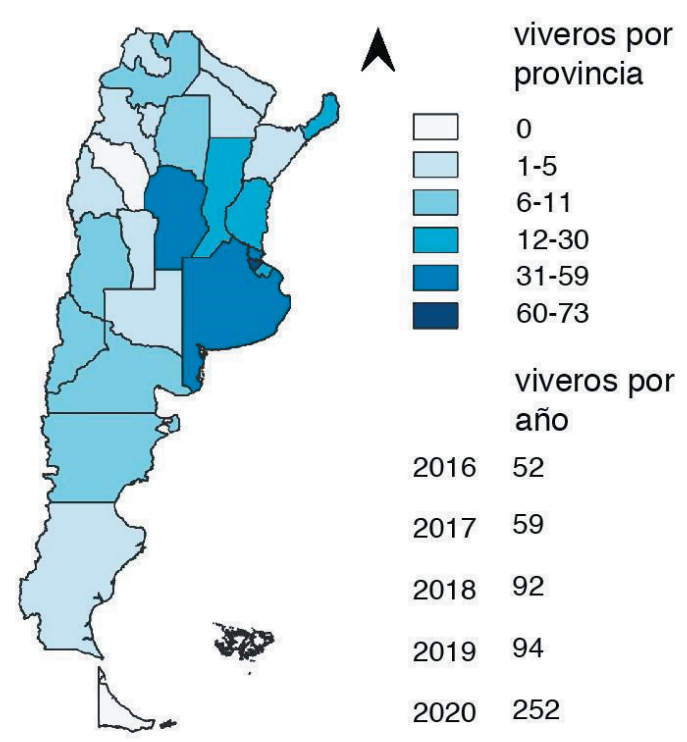

Figura 4. Cantidad de viveros por provincia y por año entre el 2016 y el 2020.

Figure 4. Number of nurseries per province per year between 2016 and 2020.

En los encuentros participaron distintas cantidades de viveros de plantas nativas. Se registró un total de 452 viveros, de los cuales 11 se ubicaban fuera de la Argentina (Material Suplementario 1). La cantidad de viveros por año aumentó de manera progresiva de 52 a 252 en el período 2016-2020 (Figura 4). El $85 \%$ de los viveros participó una única vez, un $9 \%$ participó 2 veces, un 3\% participó 3 veces, un $2 \%$ participó 4 veces y un solo vivero participó de todos los encuentros. Los viveros participantes de los encuentros procedieron principalmente de Buenos Aires y Córdoba, y también fue importante la participación de los viveros de las provincias de Santa Fe, Entre Ríos y Misiones, con algunas provincias sin viveros registrados como La Rioja y Tierra del Fuego (Figura 4). Fueron cultivadas 81 especies de plantas, pertenecientes a 27 familias, por al menos un $2 \%$ de los viveros (Tabla 1). De las especies cultivadas con más frecuencia, $32 \%$ fueron de la familia Fabaceae, 8\% Malvaceae, 7\% Bignoniaceae, $5 \%$ Verbenaceae; el resto de las familias estuvieron representadas en menos de un 5\% y sólo dos especies de las 81 pertenecieron a la clase Liliopsida (Monocotiledóneas) (Tabla 1). Las especies vegetales más frecuentemente cultivadas por los viveristas fueron Vachellia caven, Erythrina crista-galli, Celtis tala, Senna corymbosa y Enterolobium contortisiliquum (Tabla 1). La forma de vida predominante fue la de plantas leñosas, ya que entre árboles, arbustos y subarbustos sumaron un $80 \%$ de las especies más frecuentemente cultivadas. Las enredaderas o lianas representaron un $8 \%$, las hierbas un $10 \%$ y las palmeras un $1 \%$. Más del $95 \%$ de las especies cultivadas con mayor frecuencia fue perenne (Tabla 1). La distribución de estas especies se concentra en la región centro-este del país, sobre todo en las provincias de Corrientes (79\%), Entre Ríos (78\%), Buenos Aires (75\%) y Santa Fe $(74 \%)$ (Tabla 1). Las provincias del norte del país también poseen muchas de las especies cultivadas, pero en menor proporción: Salta $(71 \%)$, Chaco $(68 \%)$, Formosa (65\%), Jujuy (65\%), Misiones (64\%) y Tucumán (62\%) (Tabla $1)$. Las especies propias del sur del país son las menos frecuentemente cultivadas, de las cuales 13\% son de Río Negro, 9\% de Chubut

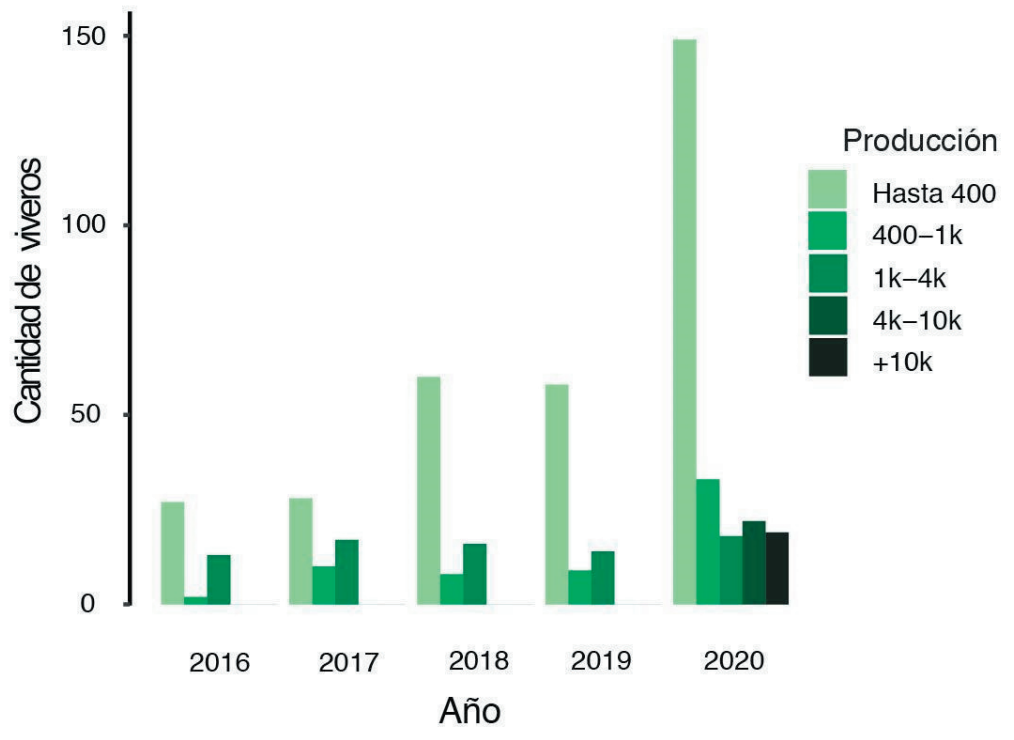

Figura 5. Cantidad de viveros participantes por año, agrupados por volumen de producción anual.

Figure 5. Number of participating nurseries per year, grouped by annual production volume. 


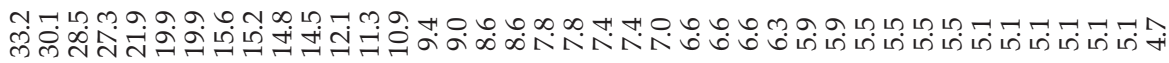

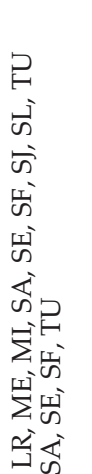

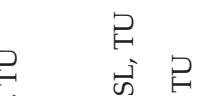

?

के के के

का में के

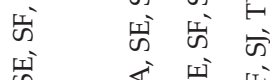

空

窉?

में के ले

के के के

岕 战 包

हे हो का

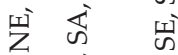

至公

हे है मुंक एँ

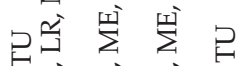

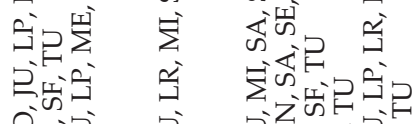

क出 㟧出它

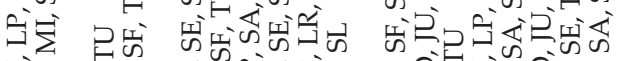

的㐫

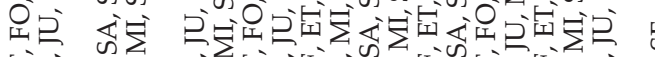

勿

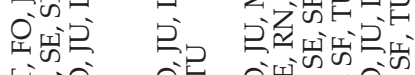

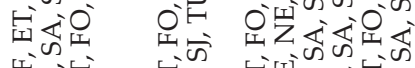

出 跑

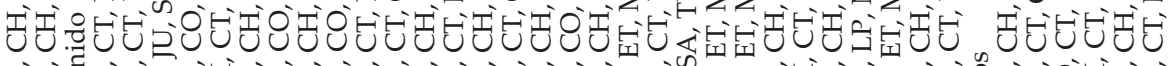

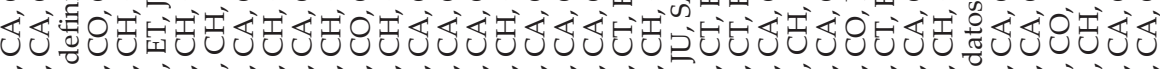

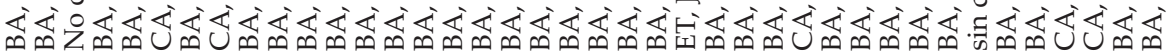

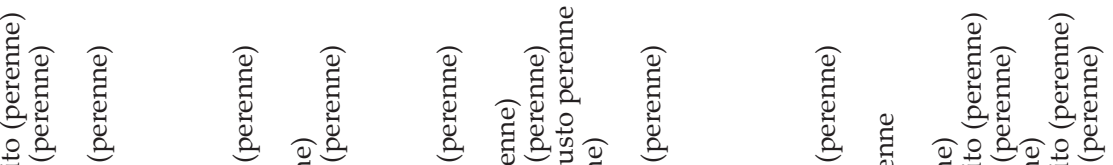
胥象

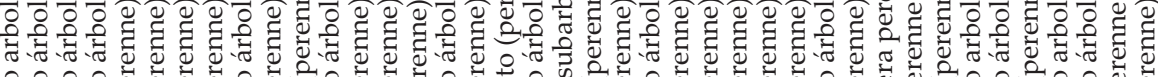

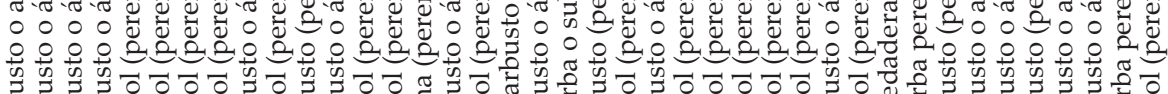

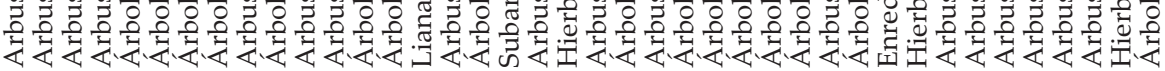
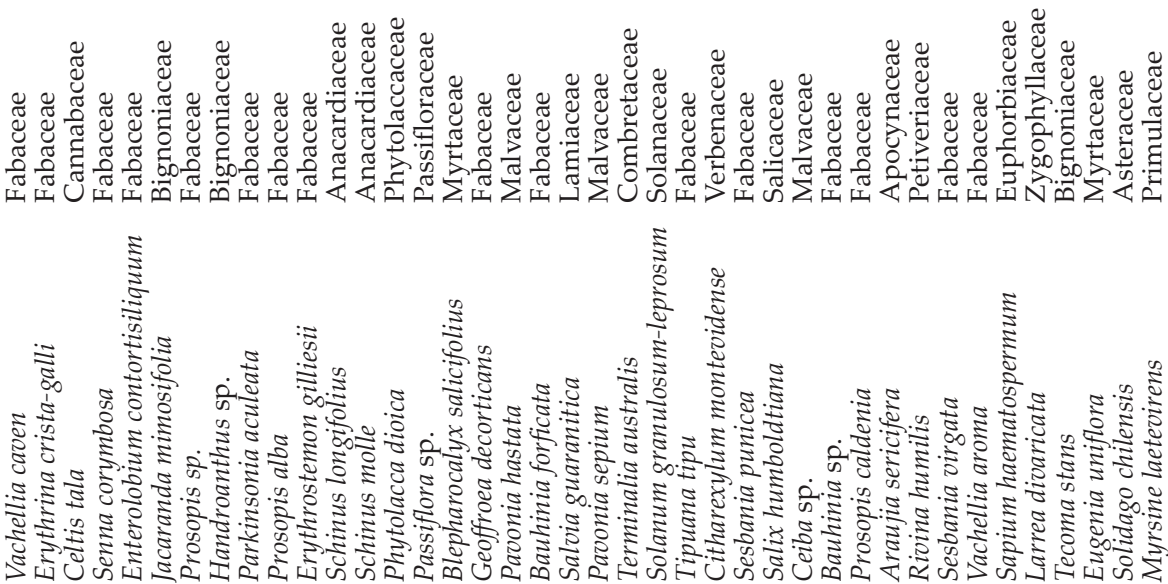


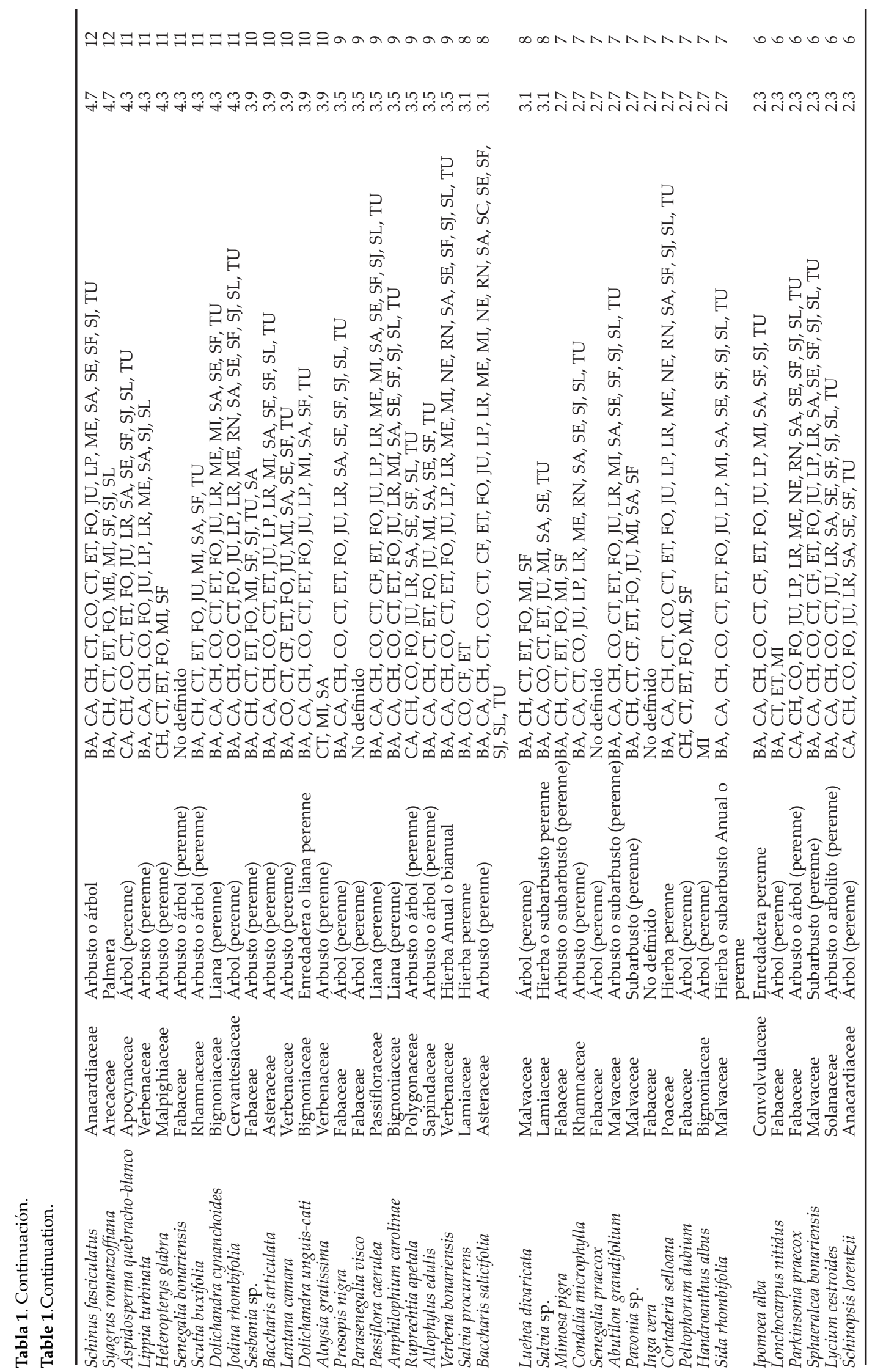


y Neuquén, 1\% de Santa Cruz y ninguna de Tierra del Fuego (Tabla 1). Todos los años, más del $50 \%$ de los viveros participantes produjeron hasta 400 plantas por año (Figura 5). Sólo en el 2020 se contó con la presencia de viveros que producen más de 4000 plantas al año (Figura 5).

\section{DisCUSIÓN}

La Red de Viveros de Plantas Nativas (REVINA) ha crecido de forma notable desde su creación, lo que refleja un interés creciente por las plantas nativas y una revalorización cultural de las mismas al difundirse su importancia para el ambiente. La REVINA es más que una red de viveros. Es una red multidisciplinaria que involucra diversos actores sociales y reúne personas de diferentes partes del país, con diferentes profesiones y pertenencias institucionales, y que poseen un interés común por las plantas nativas. La mayoría de las personas y viveros que integran la REVINA provienen de la región centro-este del país, donde se desarrolló la mayor parte de los encuentros y donde se concentra la mayor proporción de habitantes del país (INDEC 2010). Sin embargo, cuenta con la participación de representantes de todas las provincias e, incluso, de otros países. Los viveristas con viveros comerciales y no comerciales no están vinculados sólo con una determinada institución o actividad. Esta red es análoga a la Red de Restauración Ecológica de Argentina (REA), que también posee nodos a lo largo del país y convoca personas con actividades complementarias a la REVINA (i.e., mayor proporción de investigadores y funcionarios públicos que docentes) (ver Figura 3 de Pérez et al. 2018). Si bien no existen aún actividades en común entre ambas redes, sí comparten miembros, por lo que su articulación es factible y resultaría fundamental para apoyar de manera conjunta a los proyectos de restauración de la Argentina.

Los viveros de plantas nativas representan un actor social clave para los proyectos de restauración, ya que poseen el conocimiento empírico de cómo reproducir con éxito las distintas especies de plantas nativas (Handel 2017; Lacoretz et al. 2021). Así como sucede en la horticultura familiar con el cultivo de hortalizas (Pochettino et al. 2014), el valor del cultivo de plantas nativas reside en la conservación de la biodiversidad y de las prácticas culturales, y de su efecto potenciador de interrelaciones sociales. Las plantas producidas por los viveros ofrecen una aproximación a los conocimientos que los viveristas poseen sobre su cultivo, es decir, si la cultivan es porque saben cómo hacerlo o están en proceso de aprenderlo. Muchas veces, ese conocimiento es escaso en la literatura (pero ver Joseau et al. 2013; Monasterio 2016; De Luca 2020; Eynard et al. 2020), por lo que existen saberes sobre el cultivo de plantas nativas que sólo poseen los viveristas. Incluirlos en los proyectos de restauración llevaría al aprendizaje colectivo, que es el proceso de cambio social en el que las personas aprenden unos de otros y se benefician mutuamente (Ceccon et al. 2020). Además, los viveristas, al determinar qué especies de plantas cultivan, influirán en qué especies habrá disponibles como insumo de los proyectos de restauración. En este trabajo se observó que las especies de plantas cultivadas más frecuentemente por los viveros fueron de la familia Fabaceae. El resto de las familias de plantas estuvieron poco o nada representadas (como en el caso de las monocotiledóneas, con sólo dos especies registradas), lo que resulta en una muy baja equitatividad y diversidad. Las especies leñosas fueron las más frecuentemente cultivadas, y fue notoria la escasa diversidad de especies herbáceas disponibles en los viveros. Además, las especies cultivadas presentan un fuerte sesgo hacia el centroeste del país, en consonancia con el número de viveros que se concentra en esta región. Este resultado indicaría que existe una mayor producción y un mayor conocimiento sobre el cultivo de las especies de plantas de esta región que de otros lugares. Sin embargo, se debe tener en cuenta las limitaciones de estas conclusiones, ya que sólo se consideró a los viveros de plantas nativas que forman parte de la REVINA y, por lo tanto, es posible que exista producción y conocimiento sobre el cultivo de otras especies en otras regiones del país, datos no relevados en este trabajo. Para que los proyectos de restauración a nivel nacional puedan abastecerse de determinadas especies de plantas producidas por los viveros, éstos deberían representar de manera equitativa a las diferentes ecorregiones del país considerando la diversidad de especies de plantas y de formas de vidas que los conforman, y su genética, para restaurar los distintos ecosistemas.

La REVINA podría ejercer una alta influencia en los proyectos de restauración ecológica 
porque puede hacer frente a la demanda de plantas de manera conjunta, nucleando diferentes viveros. En nuestro país existen leyes y proyectos de restauración que necesitan plantas nativas como insumo. Por ejemplo, la Ley de Bosque Nativo, el Plan Nacional de Restauración de Bosques Nativos y el Plan Provincial de Recuperación del Patrimonio Ambiental-Nativas Bonaerenses (Ley Nro. 26331 2007; PNRBN 2019; PPRPA-NB 2020). Además, la Argentina está comprometida en iniciativas internacionales que implican aumentar la capacidad de restauración en la próxima década (CDB 2010; Bonn Challenge 2011; Initiative 20x20 2014). En este trabajo se observó que la mayoría de los viveros tiene una pequeña producción (i.e., menos de 400 plantas por año), aunque a lo largo de los años se sumaron viveros productores de mayor tamaño. Sin embargo, estas iniciativas demandan gran cantidad de plantas nativas como principal insumo, por lo que es necesario aumentar el volumen de producción de estos viveros e incrementar la articulación entre viveros pequeños de nuestro país para hacer frente a las grandes licitaciones de manera conjunta y planificada.

Para que esta red siga creciendo $-\mathrm{y}$ con ella la cantidad de interacciones entre actores sociales, la diversidad de especies cultivadas y el volumen de producción de plantas nativassugerimos que es necesario: 1) alentar a más viveristas a producir y vender plantas nativas. Se ha reportado que el acceso a las semillas y a la información sobre técnicas de cultivo es una limitante para producir plantas nativas (Bannister et al. 2018; León-Lobos et al. 2020; Sánchez et al. 2020). Por ello, para cumplir este objetivo proponemos que se facilite el acceso a las semillas de reservas cercanas a la ubicación de los viveros y a la información sobre su cultivo, así como también que se promueva la implementación de la Resolución Nº 318 del INASE (2018) para el registro de APSEN (Áreas Proveedoras de Semillas de Especies Nativas) (Eibl 2020); 2) mayor articulación de los viveristas con los grupos de investigación (intercambio de saberes). Se ha sugerido que para sortear los vacíos de información es necesario que exista una co-producción del conocimiento entre diferentes actores sociales (Martínez et al. 2016; Vera 2018; Norström et al. 2020); 3) asegurar el acople entre la oferta y la demanda de plantas nativas. Este desacople fue marcado como un problema para los viveristas (Handel 2017), dado que no pueden planificar de forma adecuada el volumen y la diversidad de su producción. Una posible forma de solucionarlo es crear planes de restauración definidos a largo plazo (Bannister et al. 2018), impulsados por los organismos gubernamentales (Aronson et al. 2011), y aumentar la comunicación y la interacción entre las redes de viveristas (oferta) y restauradores (demanda); 4) fomentar la educación ambiental. Es necesario explicar la importancia de las plantas nativas y de la restauración con participación de toda la sociedad (Pérez et al. 2019; Garzón et al. 2020); 5) proponer que en los próximos encuentros haya al menos un día virtual, y federalizarlos para que se pueda seguir sumando gente de todo el país y de otros países a los encuentros de la REVINA. En este sentido, es fundamental que en esta red participen actores sociales de distintos ámbitos para que se logren las articulaciones necesarias y puedan cumplirse estos desafíos; 6) relevar las actividades de los nodos y comisiones que integran la REVINA.

Con el fin de fortalecer los proyectos de restauración en la Argentina, en este proyecto se usó una perspectiva etnobotánica para estudiar la Red de Viveros de Plantas Nativas de Argentina, la comunidad de viveristas y las plantas que éstos producen. Este enfoque se puede utilizar para comprender el grado de factibilidad de los proyectos de restauración en nuestro país, que demandan plantas nativas de diversas ecorregiones en volúmenes grandes. Este acercamiento nos permitió obtener una primera lista de viveros de plantas nativas y de las especies que éstos cultivan, y discriminar algunos puntos que se pueden mejorar en vistas a fortalecer esta red de actores para poder cumplir las iniciativas de restauración del país. Es importante destacar que más allá de las propuestas de manejo que surjan desde una perspectiva ecológica, las mismas no tendrán efecto duradero a menos que estén convalidadas con el resto de la sociedad, ya que distintos actores sociales influyen sobre el ambiente (Gross 2006). Si bien se sugiere que existe la necesidad de incluir a toda la sociedad en el diseño de los proyectos de restauración, muchas veces se falla en conseguirlo (Ceccon et al. 2020). La REVINA es un espacio de interacción en el que los encargados de planificar proyectos de restauración pueden enriquecerse de la mirada de diversos actores sociales. Esperamos que la REVINA siga creciendo y que este tipo de redes se repliquen en otros países para avanzar en la restauración de ecosistemas de manera articulada. 
Agradecimientos. Agradecemos a los revisores anónimos por su interés en nuestro trabajo y por los valiosos comentarios que lo enriquecieron. Agradecemos también a D. Depalma, N. Madanes, V. Díaz Villa y C. Malavert por sus comentarios y sugerencias.

\section{REFERENCIAS}

Arenas, P. 2012. Etnobotánica en zonas áridas y semiáridas del Cono Sur de Sudamérica. CEFYBO-CONICET. Buenos Aires.

Arenas, P., and G. J. Martínez. 2012. Estudio etnobotánico en regiones áridas y semiáridas de Argentina y zonas limítrofes. Experiencias y reflexiones metodológicas de un grupo de investigación. Pp. 11-43 en Etnobotánica en zonas áridas y semiáridas del Cono Sur de Sudamérica. CEFYBO-CONICET Buenos Aires.

Aronson, J., P. H. S. Brancalion, G. Durigan, R. R. Rodrigues, V. L. Engel, M. Tabarelli, J. M. D. Torezan, S. Gandolfi, A. C. G. de Melo, P. Y. Kageyama, M. C. M. Marques, A. G. Nave, S. V. Martins, F. B. Gandara, A. Reis, L. M. Barbosa, and F. R. Scarano. 2011. What Role Should Government Regulation Play in Ecological Restoration? Ongoing Debate in São Paulo State, Brazil. Restoration Ecology 19:690-695. https://doi.org/10.1111/j.1526-100X.2011.00815.x.

Bannister, J. R., R. Vargas-Gaete, J. F. Ovalle, M. Acevedo, A. Fuentes-Ramirez, P. J. Donoso, A. Promis, and C. SmithRamírez. 2018. Major bottlenecks for the restoration of natural forests in Chile. Restoration Ecology 26:1039-1044. https://doi.org/10.1111/rec.12880.

Bardgett, R. D., and D. A. Wardle. 2010. Aboveground-belowground linkages: biotic interactions, ecosystem processes, and global change. Oxford University Press.

Bennett, B. C. 2005. Ethnobotany Education, Opportunities, and Needs in the U.S. Ethnobotany Research and Applications 3:113. https://doi.org/10.17348/era.3.0.113-122.

Bonn Challenge. 2011. Bonn Challenge. Restore our future. URL: bonnchallenge.org/about-the-goal\#commitments.

Capparelli, A., N. Hilgert, A. Ladio, V. S. Lema, C. Llano, and M. L. Pochettino. 2011. Paisajes culturales de Argentina: Pasado y presente desde las perspectivas etnobotánica y paleoetnobotánica. Revista de la Asociación Argentina de Ecología de Paisajes 2:67-79.

CDB. 2010. Convention on Biological Diversity. URL: cbd.int/countries/profile/?country=ar.

Ceccon, E., M. Méndez-Toribio, and C. Martínez-Garza. 2020. Social Participation in Forest Restoration Projects: Insights from a National Assessment in Mexico. Human Ecology 48:609-617. https://doi.org/https://doi.org/10.1007/s10745020-00178-w.

Chapin III, F. S., E. S. Zavaleta, V. T. Eviner, R. L. Naylor, P. M. Vitousek, H. L. Reynolds, D. U. Hooper, S. Lavorel, O. E. Sala, and S. E. Hobbie. 2000. Consequences of changing biodiversity. Nature 405:234-242. https://doi.org/10.1038/ 35012241.

Darwinion. 2021. Instituto de Botánica Darwinion. Catálogo de las Plantas Vasculares del Conosur Sudamérica). URL: www2.darwin.edu.ar/Proyectos/FloraArgentina/fa.htm.

De Luca, N. C. 2020. Manual de cultivo y forestación de especies nativas para el centro de Argentina. Ecoval Ediciones, Córdoba.

Díaz, S., J. Fargione, F. S. Chapin, and D. Tilman. 2006. Biodiversity loss threatens human well-being. PLoS Biology 4: 1300-1305. https://doi.org/10.1371/journal.pbio.0040277.

Dumroese, R. K., T. Luna, and T. D. Landis. 2009. Nursery Manual for Native Plants: A guide for tribal nurseries. Volumen 1: Nursery management. Agriculture Handbook. U.S. Department of Agriculture, Forest Service, Washington, D.C.

Eibl, B. 2020. Fuentes y certificación del material de propagación de especies nativas. 1er Congreso Argentino de Semillas. URL: tinyurl.com/ywkuh6mt.

Eynard, C., A. Calviño, and L. Ashworth. 2020. Cultivo de plantas nativas: propagación y viverismo de especies de Argentina central. Ecoval Ediciones, Córdoba.

Forister, M. L., V. Novotny, A. K. Panorska, L. Baje, Y. Basset, P. T. Butterill, L. Cizek, P. D. Coley, F. Dem, I. R. Diniz, P. Drozd, M. Fox, A. E. Glassmire, R. Hazen, J. Hrcek, J. P. Jahner, O. Kaman, T. J. Kozubowski, T. A. Kursar, O. T. Lewis, J. Lill, R. J. Marquis, S. E. Miller, H. C. Morais, M. Murakami, H. Nickel, N. Pardikes, R. E. Ricklefs, M. S. Singer, A. M. Smilanich, J. O. Stireman, S. Villamarín-Cortez, S. Vodka, M. Volf, D. L. Wagner, T. Walla, G. D. Weiblen, and L. A. Dyer. 2015. The global distribution of diet breadth in insect herbivores. Proceedings of the National Academy of Sciences of the United States of America 112:442-447. https://doi.org/10.1073/pnas.1423042112.

Gann, G. D., T. McDonald, B. Walder, J. Aronson, C. R. Nelson, J. Jonson, J. G. Hallett, C. Eisenberg, M. R. Guariguata, J. Liu, F. Hua, C. Echeverría, E. Gonzales, N. Shaw, K. Decleer, and K. W. Dixon. 2019. International principles and standards for the practice of ecological restoration. Second edition. Restoration Ecology 27:S1-S46. https://doi.org/ 10.1111/rec.13035.

Garzón, N. V., C. H. Rodríguez León, E. Ceccon, and D. R. Pérez. 2020. Ecological restoration-based education in the Colombian Amazon: Toward a new society-nature relationship. Restoration Ecology 28:1053-1060. https://doi.org/ 10.1111/rec.13216

Gross, M. 2006. Beyond expertise: Ecological science and the making of socially robust restoration strategies. Journal for Nature Conservation 14:172-179. https://doi.org/10.1016/j.jnc.2006.05.004.

Haase, D., and A. Davis. 2017. Developing and supporting quality nursery facilities and staff are necessary to meet global forest and landscape restoration needs. Reforesta:69-93. https://doi.org/10.21750/refor.4.06.45.

Hamilton, A., P. Shengii, J. Kessy, A. A. Khan, S. Lagos-Witte, and Z. K. Shinwari. 2003. The purposes and teaching of applied ethnobotany. United Nations Educational, Scientific and Cultural Organization (UNESCO).

Handel, S. N. 2017. Growing pains: Hopes and heartaches at native plant nurseries. Ecological Restoration 35:279-280. 
https://doi.org/10.3368/er.35.4.279.

Harrison, P. A., P. M. Berry, G. Simpson, J. R. Haslett, M. Blicharska, M. Bucur, R. Dunford, B. Egoh, M. GarciaLlorente, N. Geamănă, W. Geertsema, E. Lommelen, L. Meiresonne, and F. Turkelboom. 2014. Linkages between biodiversity attributes and ecosystem services: A systematic review. Ecosystem Services 9:191-203. https://doi.org/ 10.1016/j.ecoser.2014.05.006.

Hurrell, J. A. 2014. Urban Ethnobotany in Argentina: Theoretical advances and methodological strategies. Ethnobiology and Conservation 3:1-11. https://doi.org/10.15451/ec2014-6-3.3-1-11.

Hurrell, J. A., and U. Paulino de Albuquerque. 2012. Is Ethnobotany an Ecological Science? Steps towards a complex Ethnobotany. Ethnobiology and Conservation 1:4. https://doi.org/10.15451/ec2012-8-1.4-1-16.

INASE. 2018. Resolución EX-2018-16915944-APN-DSA\#INASE. URL: tinyurl.com/5emfjxae.

INDEC. 2010. Censo Nacional de Población, Hogares y Viviendas 2010. URL: tinyurl.com/2p8k5w7h.

Initiative 20x20. 2014. URL: tinyurl.com/ycksk5p8.

Joseau, M., M. Conles, and G. Verzino. 2013. Conservación de recursos forestales nativos de Argentina: El cultivo de plantas leñosas en vivero y a campo. Editorial Brujas, Córdoba.

Lacoretz, M. V, C. Malavert, C. Rolandi, C. Zilli, N. Madanes, and P. M. Cristiano. 2021. Caracterización de viveros de plantas nativas y su posible aporte a la restauración de los talares bonaerenses. Ecología Austral 31:242-250. https: //doi.org/10.25260/EA.21.31.2.0.1240.

León-Lobos, P., M. A. Bustamante-Sánchez, C. R. Nelson, D. Alarcón, R. Hasbún, M. Way, H. W. Pritchard, and J. J. Armesto. 2020. Lack of adequate seed supply is a major bottleneck for effective ecosystem restoration in Chile: friendly amendment to Bannister et al. (2018). Restoration Ecology 28:277-281. https://doi.org/10.1111/rec.13113.

Ley Nro. 26331. 2007. Presupuestos mínimos de protección ambiental de los bosques nativos. URL: tinyurl.com/ y74883ns.

Martínez, G., C. Romero, C. Pen, M. Villar, and P. Durando. 2016. Etnobotánica participativa en escuelas rurales de la comuna Paso Viejo (Departamento Cruz del Eje, Córdoba, Argentina). Bonplandia 25:145-162. https://doi.org/ 10.30972/bon.2521263.

MEA. 2005. Millennium Ecosystem Assessment. Ecosystems and human well-being. Island press.

Monasterio, G. 2016. Plantemos un árbol! Guía práctica para cultivar tus propios árboles. Editor Literario Rosanna Caramella, Salta.

Muiño, W. A. 2011. La etnobotánica médica del área de transición pampeano cuyana. Bonplandia 20:353-369. https: //doi.org/10.30972/bon.2021419

Norström, A. V., C. Cvitanovic, M. F. Löf, S. West, C. Wyborn, P. Balvanera, A. T. Bednarek, E. M. Bennett, R. Biggs, A. de Bremond, B. M. Campbell, J. G. Canadell, S. R. Carpenter, C. Folke, E. A. Fulton, O. Gaffney, S. Gelcich, J. B. Jouffray, M. Leach, M. Le Tissier, B. Martín-López, E. Louder, M. F. Loutre, A. M. Meadow, H. Nagendra, D. Payne, G. D. Peterson, B. Reyers, R. Scholes, C. I. Speranza, M. Spierenburg, M. Stafford-Smith, M. Tengö, S. van der Hel, I. van Putten, and H. Österblom. 2020. Principles for knowledge co-production in sustainability research. Nature Sustainability 3:182-190. https://doi.org/10.1038/s41893-019-0448-2.

Nowotny, H., P. B. Scott, and M. T. Gibbons. 2001. Re-thinking science: Knowledge and the public in an age of uncertainty. John Wiley and Sons.

Pérez, D., P. Meli, D. Renison, F. R. Barri, A. M. Beider, G. A. Burgueño, A. D. D. Galván, S. Dardelli, M. de Paz, F. M. Farinaccio, G. Papazian, M. Sirombra, and R. Torres. 2018. La Red de Restauración Ecológica de la Argentina (REA): Avances, vacíos y rumbo a seguir. Ecología Austral 28:353-360. https://doi.org/10.25260/EA.18.28.2.0.659.

Pérez, D. R., F. Del Mar González, M. E. R. Araujo, D. A. Paredes, and E. Meinardi. 2019. Restoration of society-nature relationship based on education: A model and progress in Patagonian Drylands. Ecological Restoration 37:182-191. https://doi.org/10.3368/er.37.3.182.

PNRBN. 2019. Plan Nacional de Restauración de Bosques Nativos. Resolución 267 / 2019. Secretaría de Gobierno de Ambiente y Desarrollo Sustentable. URL: tinyurl.com/4fh7jprr.

Pochettino, M. L., J. A. Hurrell, and M. M. Bonicatto. 2014. Horticultura periurbana: estudios etnobotánicos en huertos familiares y comerciales de la Argentina. Ambienta 107:2-15.

PPRPA-NB. 2020. Plan Provincial de Recuperación del Patrimonio Ambiental-Nativas Bonaerenses. OPDS, Gobierno de la Provincia de Buenos Aires. URL: tinyurl.com/2p9cw7d6.

QGIS Development Team. 2019. Quantum GIS Geographic Information System (QGIS 3.8 Zanzibar). Open Source Geospatial Foundation Project. URL: qgis.org/es/site.

R Core Team. 2021. R: A language and environment for statistical computing. R v.4.0.4. R Foundation for Statistical Computing, Vienna, Austria. URL: R-project.org.

Rosso, C. N., and G. F. Scarpa. 2019. Etnobotánica médica moqoit y su comparación con grupos criollos del Chaco argentino. Boletín de la Sociedad Argentina de Botánica 54:637-662. https://doi.org/10.31055/1851.2372.v54.n4.24868.

Rovere, A. E., S. Molares, and A. H. Ladio. 2013. Plantas utilizadas en cercos vivos de ciudades patagónicas: Aportes de la etnobotánica para la conservación. Ecologia Austral 23:165-173. https://doi.org/10.25260/EA.13.23.3.0.1171.

Sánchez, M. I., P. Aboitiz, and I. Villanova. 2020. La Red de Viveros de Plantas Nativas-REVINA, situación actual y necesidades de semillas. 1er Congreso Argentino de Semillas. URL: tinyurl.com/ywkuh6mt.

Suárez, M. E. 2014. Etnobotánica wichí del bosque xerófito en el Chaco Semiárido salteño. Editorial autores de Argentina.

Vera, C. 2018. Farmers transformed how we investigate climate. Nature 562:9. https://doi.org/10.1038/d41586-01806856-6. 